PII: S0735-1933(01)00246-9

\title{
EFFECT OF MOVING DISTANCE OF A MOVING BLOCK ON HEAT TRANSFER IN A CHANNEL FLOW
}

\author{
Wu-Shung Fu, Shih-Fa Chen and Ke-Nan Wang \\ Department of Mechanical Engineering \\ National Chiao Tung University \\ Hsinchu, Taiwan 30010 \\ R.O.C.
}

(Communicated by J.P. Hartnett and W.J. Minkowycz)

\begin{abstract}
A numerical investigation of heat transfer rate of an insulated block moving on a heated surface in a channel was studied. The study mainly investigated the effect of block moving distance on the heat transfer rate of heated surface. This subject belongs to a kind of moving boundary problems, and the modified Arbitrary Lagrangian Eulerian method is suitable for solving this subject. The results show that the block moving distance affects the flow and thermal fields remarkably. The heat transfer rate of heated surface increases proportionally to the increment of the block moving distance, when the block moving distance is larger than a critical value. 2001 Eisevier Science Ltd
\end{abstract}

\section{Introduction}

Up to now, numerous methods have been proposed to enhance heat transfer rate of a channel. However, accompanying with the progress of semiconductor technology, the smaller 
and more compact devices are produced indefatigably. How to increase the heat transfer rate of the heated devices becomes a very important issue.

A method of a channel or pipe installed with ribs is often used to enhance the heat transfer rate of the channel or pipe. Lots of studies such as Sparrow et al. [1] and Braaten and Patankar [2] investigated this issue, and the results showed that the ribs disturbed the flow field and enlarged the heat transfer area that caused the increment of the heat transfer rate. Liou et al. [3] investigated experimentally the variations of the mean velocity and turbulence intensity of the channel flow by mounting two pairs of turbulence promoters in tandem, and showed that the rib pitch-to-height ratio affected the phenomena of separation, reattachment and heat transfer rate. Lin and Hung [4] studied the transient forced convection in a vertical rib-heated channel with a turbulence promoter, and found that the utilization of a turbulence promoter could effectively improve the heat transfer performance in the fully developed region. Iyer and Kakac [5] investigated heat transfer in a channel with periodic grooves to simulate electronic components numerically, and found that the fluid developed a large-amplitude, time-periodic and nonlinear oscillation beyond a critical Reynolds number. The oscillations enhanced the heat transfer rate of the heated walls.

From the above literature, due to the existence of the ribs, the phenomena of separation and reattachment occurred in the flow field, which caused the heat transfer in the circulation zone to be complex and small. Then, the increment of heat transfer rate of the channel flow seems to have limitation. Fu et al. [6] investigated numerically the forced convection heat transfer in a channel with a moving block. The results showed that the moving block destroyed the boundary layer on the heated surface, and the new boundary layer reformed right after the moving block passing through. Based upon the reformation of new boundary layer, the maximum increment of heat transfer rate was about $98 \%$. However, due to the limitation of contents of Fu et al. [6], the results about the effect of the block moving distance on the heat transfer of heated surface are 
incomplete.

Therefore, the aim of the study is to continue the investigation of previous study [6] of a block moving back and forth on a heated surface in a channel. The effect of the block moving distance on the heat transfer of heated surface is mainly focused in the study. The subject mentioned above belongs to a kind of moving boundary problems, and the Arbitrary Lagrangian Eulerian (ALE) method modified by Fu and Yang [7] is suitably adopted to solve this problem. The results show that the increment of the heat transfer rate of heated surface is proportion to the block moving distance, when the block moving distance is larger than a critical value

\section{Analysis}

The physical model is shown in Fig. 1. A horizontal channel with length $L$ and height $H$ is used to simulate the two-dimensional flow channel. The fully developed air, of which the temperature is $T_{0}$, flows into the channel from the inlet $\mathrm{AB}$ and out from the outlet $\mathrm{CD}$. The temperature of the heated surface EF with length $l_{\mathrm{h}}$ is $T_{\mathrm{h}}$ which is higher than $T_{0}$. The other surfaces of the channel (surfaces $\mathrm{AD}, \mathrm{BE}$ and $\mathrm{FC}$ ) are insulated. An insulated moving block GHIJ with height $h$ and width $b$ is set on the heated surface and moves back and forth from the positions $\mathrm{M}$ to $\mathrm{N}$ with a constant velocity $u_{\mathrm{b}}$.

Initially $(\tau=0)$, the block is stationary at the middle of the heated surface, and the flow field in the channel is steady. As the time $\tau>0$, the block starts to move along the MG line on the heated surface with the constant velocity $-u_{\mathrm{b}}$. When the left surface GH of the block reaches the left point $\mathrm{M}$, the block turns back instantly and moves to the right side with the constant velocity $u_{\mathrm{b}}$. Similarly, when the right surface $\mathrm{IJ}$ of the block reaches the right point $\mathrm{N}$, the block turns back immediately and moves to the left side with the constant velocity $-\boldsymbol{u}_{\mathrm{b}}$. The reciprocation motion of the block mentioned above is continuously executed on the region MN. 
Consequently, the behavior of both the flow and thermal fields becomes time-dependent and can be categorized into a kind of moving boundary problem. As a result, the ALE method is properly utilized to analyze this problem.

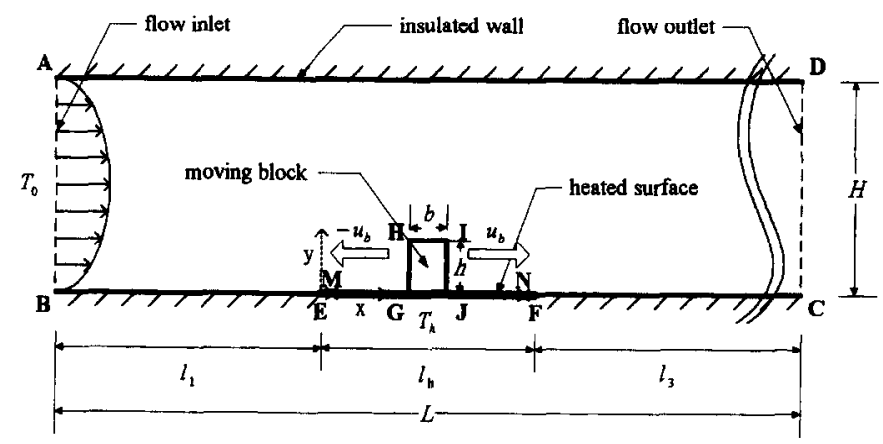

FIG. 1

Physical model.

To simplify the analysis, the following assumptions and the dimensionless variables are made.

1. The flow field is two-dimensional and laminar.

2. The fluid is Newtonian and incompressible.

3. The fluid properties are constant.

4. The no-slip condition is held on the interface between the fluid and block.

5. The effect of gravity is neglected.

$\mathrm{X}=\frac{x}{H}, \quad \mathrm{Y}=\frac{y}{H}, \quad \mathrm{R}=\frac{r}{H}, \quad \mathrm{U}=\frac{u}{\bar{u}_{0}}, \quad \mathrm{~V}=\frac{v}{\bar{u}_{0}}$,

$\hat{\mathrm{U}}=\frac{\hat{u}}{\bar{u}_{0}}, \quad \mathrm{U}_{\mathrm{b}}=\frac{u_{\mathrm{b}}}{\bar{u}_{0}}, \quad \mathrm{P}=\frac{p-p_{\infty}}{\rho \bar{u}_{0}^{2}}, \quad \tau=\frac{t \bar{u}_{0}}{H}$,

$\theta=\frac{T-T_{0}}{T_{h}-T_{0}}, \quad \operatorname{Re}=\frac{\bar{u}_{0} H}{v}, \quad \operatorname{Pr}=\frac{v}{\alpha}$, 
where $\bar{u}_{0}$ is the mean velocity of the inlet velocity distribution, and $\hat{u}$ is mesh velocity.

Based upon the above assumptions and dimensionless variables, the dimensionless ALE governing equations (2)-(5) are expressed as the following equations:

Continuity equation

$\frac{\partial \mathrm{U}}{\partial \mathrm{X}}+\frac{\partial \mathrm{V}}{\partial \mathrm{Y}}=0$

Momentum equations

$$
\begin{aligned}
& \frac{\partial \mathrm{U}}{\partial \tau}+(\mathrm{U}-\hat{\mathrm{U}}) \frac{\partial \mathrm{U}}{\partial \mathrm{X}}+\mathrm{V} \frac{\partial \mathrm{U}}{\partial \mathrm{Y}}=-\frac{\partial \mathrm{P}}{\partial \mathrm{X}}+\frac{1}{\mathbf{R e}}\left(\frac{\partial^{2} \mathrm{U}}{\partial \mathrm{X}^{2}}+\frac{\partial^{2} \mathrm{U}}{\partial \mathrm{Y}^{2}}\right) \\
& \frac{\partial \mathrm{V}}{\partial \tau}+(\mathrm{U}-\hat{\mathrm{U}}) \frac{\partial \mathrm{V}}{\partial \mathrm{X}}+\mathrm{V} \frac{\partial \mathrm{V}}{\partial \mathrm{Y}}=-\frac{\partial \mathrm{P}}{\partial \mathrm{Y}}+\frac{1}{\operatorname{Re}}\left(\frac{\partial^{2} \mathrm{~V}}{\partial \mathrm{X}^{2}}+\frac{\partial^{2} \mathrm{~V}}{\partial \mathrm{Y}^{2}}\right)
\end{aligned}
$$

Energy equation

$$
\frac{\partial \theta}{\partial \tau}+(\mathrm{U}-\hat{\mathrm{U}}) \frac{\partial \theta}{\partial \mathrm{X}}+\mathrm{V} \frac{\partial \theta}{\partial \mathrm{Y}}=\frac{1}{\operatorname{PrRe}}\left(\frac{\partial^{2} \theta}{\partial \mathrm{X}^{2}}+\frac{\partial^{2} \theta}{\partial \mathrm{Y}^{2}}\right)
$$

As $\tau>0$, the boundary conditions are as follows:

On the surfaces $\mathrm{AD}, \mathrm{BE}$ and $\mathrm{FC}, \mathrm{U}=\mathrm{V}=0, \partial \theta / \partial \mathrm{n}=0$;

On the inlet surface $A B, U=-6\left(Y^{2}-Y\right), V=0, \theta=0$;

On the heated surfaces $E G$ and $J F, U=V=0, \theta=1$;

On the outlet surface $C D, \partial U / \partial n=\partial V / \partial n=\partial \theta / \partial n=0$;

On the surfaces of the block $\mathrm{GH}, \mathrm{HI}$ and $\mathrm{IJ}, \mathrm{U}=\mathrm{U}_{\mathrm{b}}, \mathrm{V}=0, \partial \theta / \partial \mathrm{n}=0$.

\section{Numerical Method}

A Galerkin finite element method with moving mesh is adopted to solve the governing equations (2)-(5). A backward scheme is used to deal with the time differential terms in the governing equations. A penalty function and Newton-Raphson iteration algorithm are utilized to reduce the pressure and nonlinear terms in the momentum equations, respectively. The velocity 
and temperature terms are expressed as quadrilateral and nine-node quadratic isoparametric elements. The discretization processes of the governing equations are similar to those used in $\mathrm{Fu}$ and Yang [7].

The value of penalty parameter used in the present study is $10^{6}$, and the frontal method solver is utilized to solve the governing equations. The value of Prandtl number is 0.71 for air. The mesh velocity $\hat{\mathrm{U}}$ is linear distribution and inverse proportion to the distance between the node and the moving block

The computational mesh and the non-dimensional time intervals $\Delta \tau$ are tested under $\operatorname{Re}=500$ and $\mathrm{U}_{\mathrm{b}}=1.0$. For economizing computational time, the length of the inlet channel $l_{1}$, the heated surface $l_{\mathrm{h}}$, the outlet channel $l_{3}$, the width $b$ and the height $h$ of the block are chosen as $3 H, H, 20 H, 0.1 H$ and $0.2 H$, respectively, and the mesh with $114 \times 34$ elements is chosen for computations. In addition, the value of non-dimensional time intervals $\Delta \tau$ is chosen as $2 \times 10^{-3}$ for all block moving distances.

\section{Results and Discussion}

As the block moved on the heated surface, the boundary layer on the heated surface before the moving block was destroyed and reformed immediately as the block passed through. As a result, the heat transfer rate of the heated surface increased remarkably as shown in the previous study [6]. However, the results of the effect of block moving distance (R) on the heat transfer of heated surface are inadequate in the above study. Then, in the present study, the block moving distance varying from 0 to 0.8 are investigated. For clearly indicating the phenomena around the block, only the flow and thermal fields close to the moving block are illustrated in the following figures. 


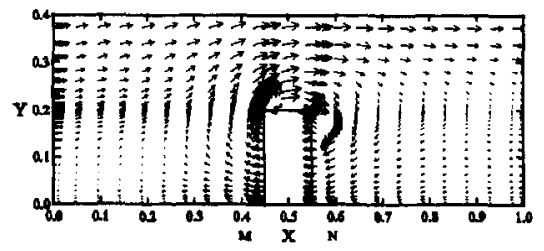

(a1) $\tau=2.60$

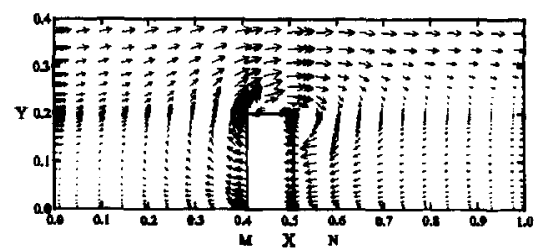

(a2) $\tau=2.65$

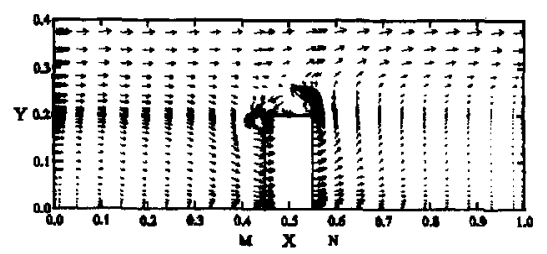

(a3) $\tau=2.70$

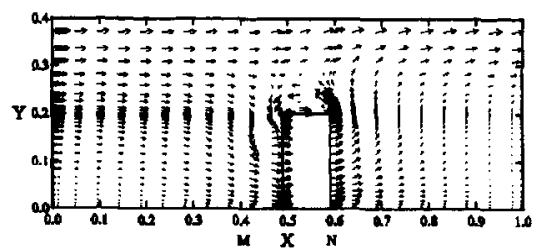

(a4) $\tau=2.75$

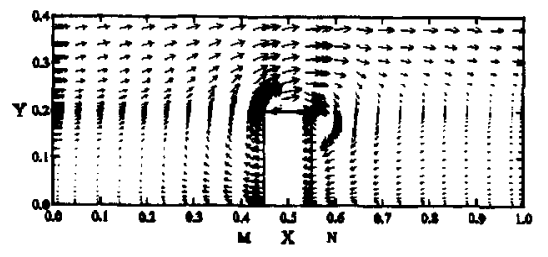

(a1) $\tau=2.80$

(a) $\mathrm{R}=0.1$

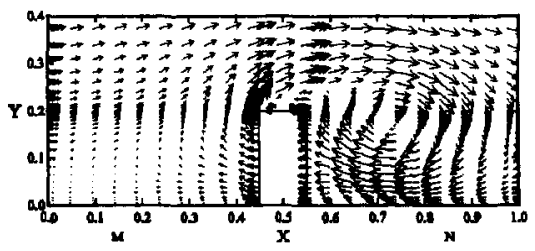

(b1) $\tau=7.2$

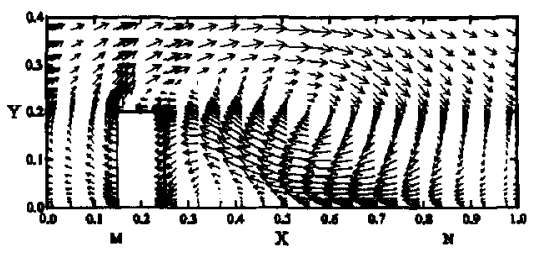

(b2) $\tau=7.5$

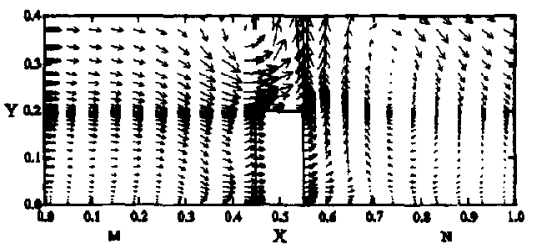

(b3) $\tau-7.8$

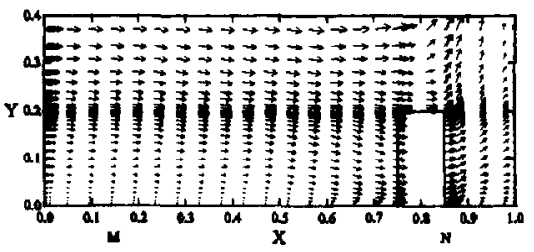

(b4) $\tau=8.1$

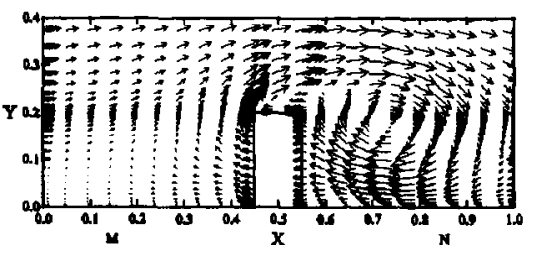

(b5) $\tau=8.4$

(b) $\mathrm{R}=0.6$

FIG. 2

The velocity vectors near the moving block for (a) $R=0.1$ and $(b) R=0.6$ under $R e=500$ and $\mathrm{U}_{\mathrm{b}}=1.0$ cases. 


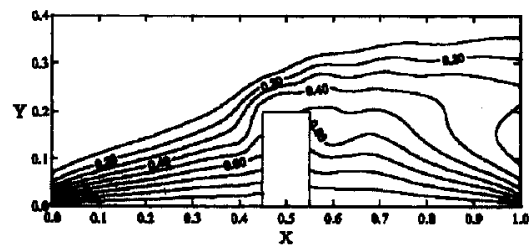

(al) $\tau=2.60$

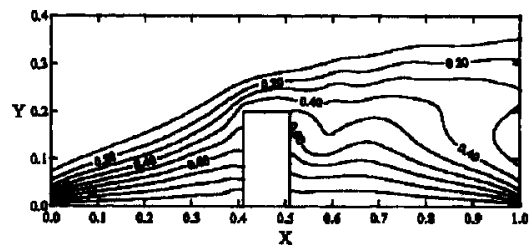

(a2) $\tau=2.65$

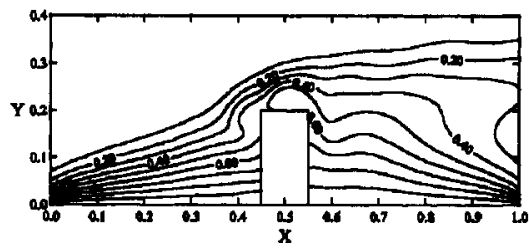

(a3) $\tau=2.70$

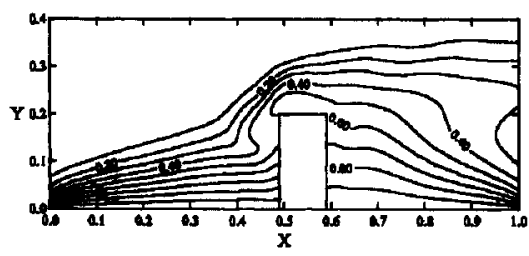

(a4) $\tau=2.75$

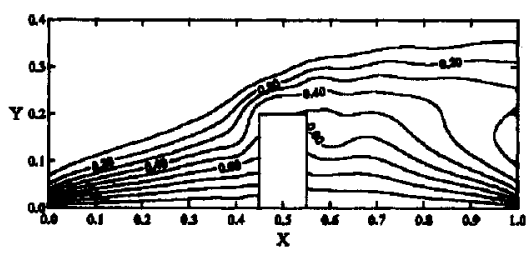

(a5) $\tau=2.80$

(a) $\mathrm{R}=0.1$

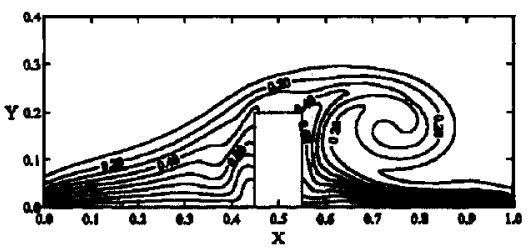

(b1) $\tau=7.2$

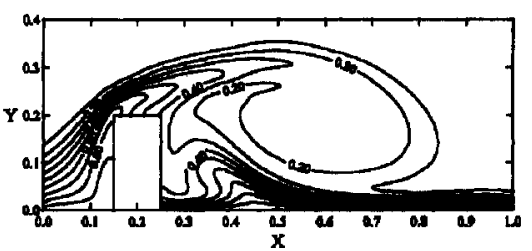

(b2) $\tau=7.5$

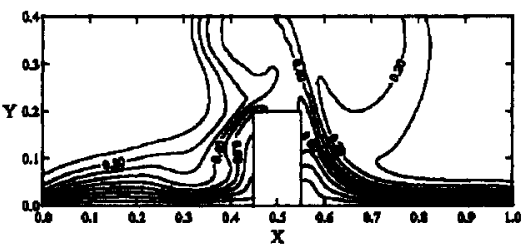

(b3) $\tau=7.8$

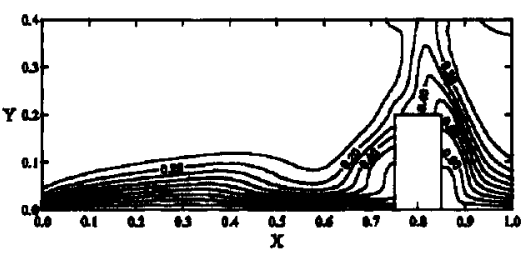

(b4) $\tau=8.1$

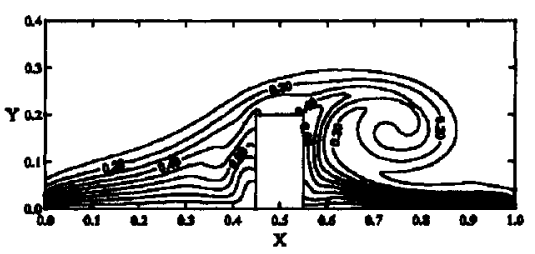

(b5) $\tau=8.4$

(b) $\mathrm{R}=0.6$

\section{FIG. 3}

The isothermal lines near the moving block for $(a) R=0.1$ and $(b) R=0.6$ under $R e=500$ and $U_{b}=1.0$ cases. 


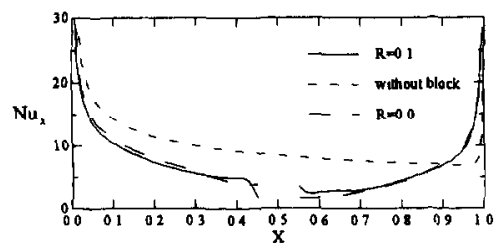

(a1) $\tau=2.60$

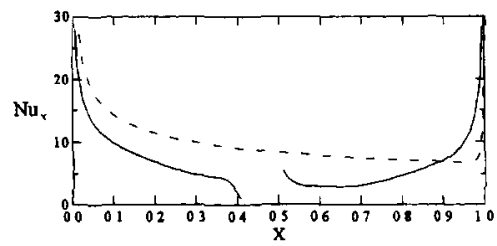

(a2) $\tau=2.65$

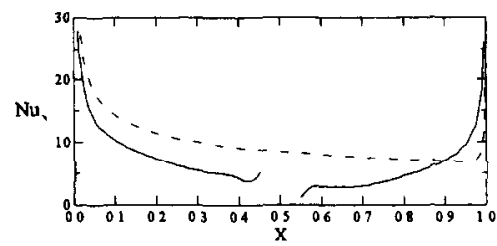

(a3) $\tau=2.70$

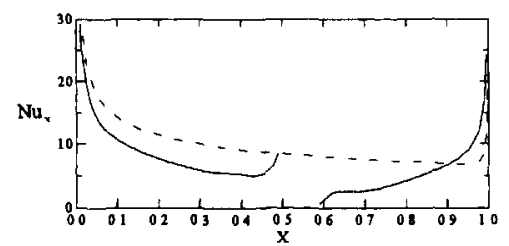

(a4) $\tau=2.75$

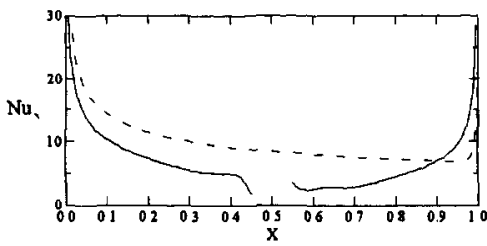

(a5) $\tau=2.80$

(a) $\mathrm{R}=0.1$

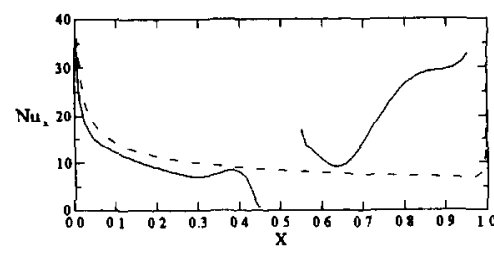

(b1) $\tau=7.2$

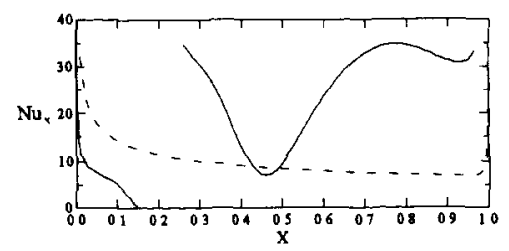

(b2) $\tau=7.5$

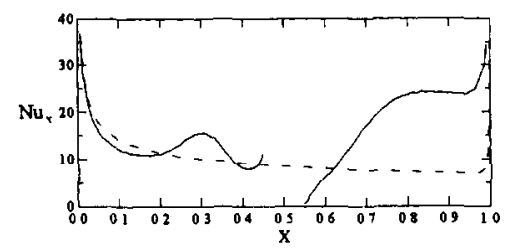

(b3) $\tau=7.8$

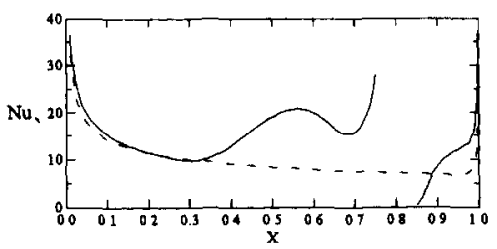

(b4) $\tau=8.1$

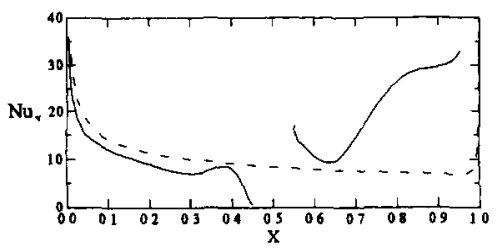

(b5) $\tau=8.4$

(b) $R=0.6$

FIG. 4

The distributions of local Nusselt number on the heated surface for $(a) R=01$ and (b) $R=0.6$ under $\operatorname{Re}=500$ and $U_{b}=1.0$ cases. 
The variations of the velocities, isothermal lines and local Nusselt numbers $\mathrm{Nu}_{\mathrm{x}}$ for $R=0.1$ and 0.6 under $R e=500$, and $U_{b}=1.0$ are indicated in FIGs. 2-4, respectively. The local Nusselt number $\mathrm{Nu}_{\mathrm{x}}$ is defined as the follow equation.

$\mathrm{Nu}_{\mathrm{x}}=-\left.\frac{\partial \theta}{\partial \mathrm{Y}}\right|_{\mathrm{Y}=0}$.

These phenomena shown in Figs. 2-4 are in a certain period of total reciprocation motions. In this period, the variations of the velocity and thermal fields become cyclic.

In FIGs. 2(a1), (a2) and (b1), (b2), the block moves from the center position to the left point $M$ of the heated surface. The direction of the moving block is different from the mainstream. The velocities of the fluids close to the moving block are much larger than those of the fluids far away from the moving block, and the original velocity boundary is destroyed by the moving block. On the right surface of the block, the fluid complements the vacant space induced by the moving block instantly, and a new velocity boundary layer reforms after the moving block. In $R=0.6$ case (FIGs. 2(b1) and (b2)), the velocities of fluids are larger than those of $R=0.1$ case (FIGs. 2(a1) and (a2)), due to the increment of block moving distance. And, a recirculation zone behind the block is observed in $\mathrm{R}=0.6$ case.

The moving block turns back immediately, when the left surface of the block reaches the left point M. In FIGs. 2(a3), (a4) and (b3), (b4), the block moves from the central position to the right point $\mathrm{N}$ of the heated surface. The direction of fluid behind the moving block is the same as that of the mainstream, and then more fluids flow toward the heated surface and the boundary layer reforms right behind the moving block. Before the block, the fluids are pressed by the moving block and flow rightward. However, due to the formation of the recirculation zone on the right side of the block, some fluids flow leftward. Then, the two streams of fluids mentioned above with different directions interact with each other that causes the interflow to flow upward.

In FIGs. 2(a5) and (b5), the block moves leftward and reaches the central position of the heated surface again. The flow fields at this moment are very similar to those shown in FIGs 
2(a l) and (bl), that indicates the variation of flow field to be cyclic.

FIG. 3 shows the distributions of isothermal lines at the same condition shown in FIG.2 The thermal fields are usually corresponding to the characteristics of the flow fields, and the thermal boundary layer on the heated surface is destroyed by the moving block and reforms right after as the block passes through. The isothermal lines shown in FIGs. 3(a1) (a5) are similar, because the region affected by the moving block is small in $\mathrm{R}=0.1$ case. Oppositely in FIGs. 3(b1) (b5), the variations of isothermal lines are drastic and the distribution of isothermal lines are dense, which causes the heat transfer of the heated surface to increase remarkably.

The distributions of local Nusselt numbers $\mathrm{Nu}_{\mathrm{x}}$ shown in FIG. 4 are under the same condition as shown in FIG. 3. The dash lines shown in Fig. 4 indicate the distributions of the local Nusselt number of the heated surface when the channel is without the moving block. Due to the insulation of the block, the distributions of the local Nusselt number shown by solid lines are interrupted. In FIGs. 4(a1), (a2), and (b1), (b2), the direction of block is different from that of the mainstream, and the block becomes an obstructer. As a result, the local Nusselt numbers before the moving block are lower than those without block on the heated surface. Oppositely, a new thermal boundary layer reforms behind the moving block, which enlarges the local Nusselt number apparently. When the block moves rightward (shown in FIGs. 4(a3), (a4) and (b3), (b4)), more fluids induced by the block, and thermal boundary layer reforms near the left surface of the block. Consequently, the local Nusselt numbers increase remarkably behind the block.

The variations of average Nusselt numbers $\overline{\mathrm{Nu}_{\mathrm{x}}}$ of the heated surface of a certain cycle are shown in FIG. 5. The definition of the average Nusselt number $\overline{\mathrm{Nu}_{\mathrm{x}}}$ is shown in the following equation,

$$
\overline{\mathrm{Nu}}=\int_{\text {heat plate }} \mathrm{Nu}_{\mathrm{x}} \mathrm{dX}
$$

where the dimensionless length of the heated surface equals to 1. 


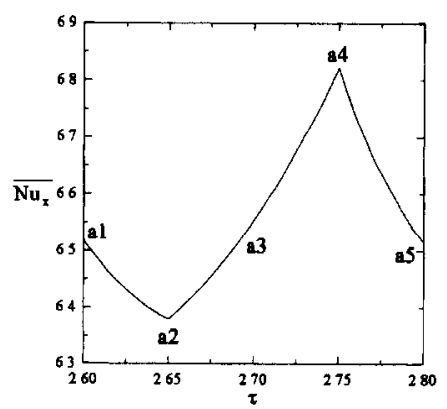

(a) $\mathrm{R}=0.1$

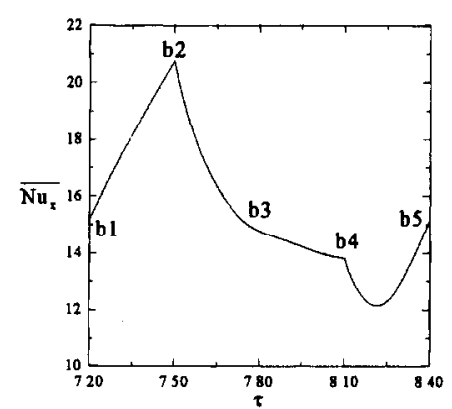

(b) $\mathrm{R}=0.6$

FIG. 5

The variations of the average Nusselt number $\overline{\mathrm{Nu}_{\mathrm{x}}}$ with $\tau$ for (a) $\mathrm{R}=0.1$, (b) $\mathrm{R}=0.6$ under $\operatorname{Re}=500$ and $U_{b}=1.0$ cases.

As the block moves leftward in case $\mathrm{R}=0.1$ shown in FIGs. 2(a1) and (a2), the new thermal boundary layer reforms right after the moving block, and the moving block prevents the mainstream to flow toward the heated surface. As a result, the average Nusselt numbers decrease in this duration (from 'al' to 'a2' shown in FIG 5(a)). When the block moves rightward from ' $a 2$ ' to ' $a 4$ ' shown in FIG. 5(a), the moving block induces more fluids to flow toward the heated surface, and the local Nusselt numbers becomes large behind the block. And, the average Nusselt number increases.

In $\mathrm{R}=0.6$ case shown in FIG. $5(\mathrm{~b})$, the region affected by the moving block is large, and the recirculation zone behind the moving block induces more low temperature fluids toward the heated surface. As a result, the average Nusselt number increase continuously when the block moves leftward (the time from 7.2 to 7.5 in FIG. 5(b)). As the block moves rightward from left point $\mathrm{M}$ to right point $\mathrm{N}$, the directions and velocities of the mainstream and those of the moving block are the same, which causes the phenomena of fluids induced by the moving block to be not 
remarkable. Then, the local Nusselt number decreases before the block, and the average Nusselt number decreases continuously (from 'b2' to ' $b 4$ ' in FIG. 5(b)). As the block passes through the central position of the heated surface and moves continuously to the right, more fluids flow toward the heated surface, and the local Nusselt number behind the block becomes large. As a result, the decrement of the average Nusselt number slows down (from 'b3' to 'b4' in FIG. 5(b)). When the block begins to move leftward from the right point $\mathrm{N}$, the fluid induced by the block is weak behind the block. Then, the average Nusselt number still decreases. Afterward, the new boundary layer region behind the block becomes larger, and the recirculation zone induces more cold fluids to flow toward the heated surface. Consequently, the average Nusselt number starts to increase again. When the block is at the central position of the heated surface, the variations of the averaged Nusselt number become periodic.

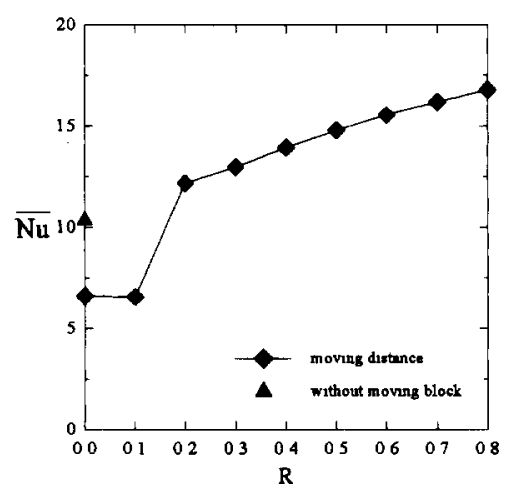

FIG. 6

The variations of time average Nusselt number per unit cycle $\overline{\mathrm{Nu}}$ with block moving distance $R$ under $\operatorname{Re}=500$ and $U_{b}=1.0$ cases.

FIG. 6 shows the variations of average Nusselt numbers per unit cycle $\overline{\mathrm{Nu}}$ on the heated surface for all cases from $R=0$ to $R=0.8$ under $R e=500$, and $U_{b}=1.0$. The average Nusselt number per unit cycle is defined as the following equation, 
$\overline{\mathrm{Nu}}=\frac{1}{\mathrm{~T}} \int_{\mathrm{T}} \overline{\mathrm{Nu}_{\mathrm{x}}} \mathrm{d} \tau$

where $\mathrm{T}$ is the dimensionless time per unit cycle. The sign " shown in these figures indicate the average Nusselt number per unit cycle $\overline{\mathrm{Nu}}$, and the sign " $\mathbf{\Delta}$ " represent the average Nusselt number of the heated surface when the channel is without the moving block

Shown in FIG. 6 , the region affected by the moving block is small in $\mathrm{R}=0.1$ case, and the heated transfer rate increases slightly. As the block moving distance increases, the effect of the block movement on the heat transfer rate of the heated surface is remarkable. As a result, the average Nusselt number per unit cycle increases continuously.

\section{Conclusions}

A numerical investigation of the effect of block moving distance on heat transfer of a channel is studied. The main conclusions can be summarized as follows

(1) The block moving distance affects the flow and thermal fields near the block and heated surface remarkably.

(2) The increment of heat transfer rate of the heated surface is proportional to the block moving distance, when the block moving distance is larger than a critical value.

\section{Acknowledgment}

The support of this work by National Science Council, Taiwan, R.O.C. under contract NSC89-2212-E-009-072 is gratefully acknowledgment 


\section{Nomenclature}

$b$ dimensional width of the block

$h$ dimensional height of the block

\section{Greek symbols}

$H$ dimensional height of the channel

$\alpha \quad$ thermal diffusivity

$L \quad$ dimensional length of the channel

$\delta$ dimensional boundary layer thickness

$l$ dimensional length of the wall

$\theta$ dimensionless temperature

n the normal vector of surface

$\lambda$ penalty parameter

$\mathrm{Nu}$ Nusselt number

$v \quad$ kinematic viscosity

$p \quad$ dimensional pressure

$\rho$ dimensional density

$p_{\infty}$ dimensional reference pressure

$\tau$ dimensionless time

P dimensionless pressure

Pr Prandtl number

\section{Subscripts}

$r$ dimensional block moving distance

0 aiflow inlet

R dimensionless block moving distance

1 inlet channel

Re Reynolds number

3 outlet channel

$t$ dimensional time

b block

$T$ dimensional temperature

h heated surface

$u, v$ dimensional velocities in $\mathrm{x}$ and $\mathrm{y}$

x local

directions

$\mathrm{U}, \mathrm{V}$ dimensionless velocities in $\mathrm{X}$ and $\mathrm{Y}$

\section{Superscripts}

directions

- mean value

$x, y$ dimensional Cartesian coordinates

$\wedge$ grid

$\mathrm{X}, \mathrm{Y}$ dimensionless Cartesian coordinates 


\section{References}

1. E.M. Sparrow, J.E. Nithammer and A. Chaboki, Int. J. Heat Mass Transfer, 25, 961 (1982).

2. G. Bergeles and N. Athanassiadis, J. Fluids Eng. Trans. ASME. 105, 461 (1983).

3. T.M. Liou, Y. Chang, D.W. Hwang, J. Fluids Eng. Trans. ASME. 112, 302 (1990)

4. H.H. Lin and Y.H. Hung, Int. J. Heat Mass Transfer, 36, 1553 (1993).

5. R.S. Iyer, S. Kakac and K.Y. Fung, J. Thermophysics Heat Transfer, 11, 437 (1997).

6. W. S. Fu, W. W. Ke and K. N. Wang, Int. J. Heat Mass Transfer, accepted.

7. W. S. Fu and S.J. Yang, Heat Mass Transfer, 36, 257 (2000). 